\title{
The Effects of Maternal Hyperthyroidism on Histologic Changes in Parietal Lobe in Rat Embryos
}

\author{
Fatemeh Mirsaf, ${ }^{1}$ Gholamreza Kaka, ${ }^{2,}$ and Mahnaz Azarnia ${ }^{1}$ \\ ${ }^{1}$ Department of Animal Biology, Faculty of Biological Sciences, Islamic Azad University, North Tehran Branch, Tehran, Iran \\ ${ }^{2}$ Neuroscience Research Center, Baqiyatallah University of Medical Sciences, Tehran, Iran \\ "Corresponding author: Gholamreza Kaka, PhD, Neuroscience Research Center, Baqiyatallah University of Medical Sciences, Aghdasie, Artesh Blvd, Artesh Sq, Tehran, Iran. \\ Tel/Fax: +98-2126127286, E-mail: gh_kaka@yahoo.com
}

Received 2016 December 07; Revised 2017 January 09; Accepted 2017 May 08.

\begin{abstract}
Background: Maternal hyperthyroidism causes developmental defects on the nervous system of fetuses.

Objectives: The present study was designed to study the effects of maternal hyperthyroidism on the development of the parietal lobe in the brain of rat embryos.

Methods: In this experimental study, thirty Sprague-Dawley rats were randomly divided into three groups. The control group received no injections, the sham group received intraperitoneal injections of distilled water solution containing salt and polysorbate (solvent of levothyroxine), and the experimental group received once-daily, intraperitoneal injections of $0.5 \mathrm{mg} / \mathrm{kg}$ levothyroxine for a 10-day period to become hyperthyroid rats. The hyperthyroid rats were then mated, and all pregnant rats were killed on the 20 th day of gestation. Fetuses were removed, fixed, and processed for histological procedures. The fetuses were sagitally sectioned at $5 \mu$ thickness and stained with hematoxylin-eosin ( $\mathrm{H}$ and $\mathrm{E}$ ) technique. The sections were examined using a light microscope and Motic software.

Results: The results showed no significant difference in the studied variables between the sham and control groups. A significantly increase in body weight and a significant decrease in crown-rump length of embryos was observed in the experimental group when compared to the control group. The mean total thickness of the parietal cortex, ventricular layer, and intermediate layer of embryos showed a significant decrease in the experimental group compared to the control and sham groups. The mean number of cells also showed a significant decrease in the intermediate and ventricular layers in the experimental group compared to the control and sham groups.

Conclusions: This study showed that maternal hyperthyroidism leads to a reduction in development of the parietal cortex in embryos. Maternal hyperthyroidism can disturb the growth and development of embryos.
\end{abstract}

Keywords: Hyperthyroid, Parietal Lobe, Fetus, Rat

\section{Background}

One of the most important stages of embryonic growth is organogenesis. The central nervous system (CNS) in humans originates in the middle of the third week in the form of a neural plate from the ectoderm, and after the edges of the plate fold, the neural folds come together in the middle line and form the neural tube. The head (crown) end of the tube is closed in about 25 days and the tail (rump) end is closed in 27 days. The result is the formation of the tube-shaped structure of the CNS of which its head end is wide (brain) and its tail end is long (spine). The growth of brain begins in the fifth week of pregnancy, and following it, the cerebral hemispheres are formed on the sides [1]. Organogenesis is intensively impacted by different environmental factors such as physical and chemical factors. Among the chemical factors; it is possible to refer to hormones and medicines. If a mother is not treated on time, it will be associated with the emergence of abnor- mality, acute consequences in the central neural system, preterm delivery, or increased embryo mortality. In addition, each factor under the impact of the dose consumed by the mother and the time of impact has different effects on the embryo [1-3]. Any kind of deficiency in the closing of the neural tube will bring about deficiencies in the embryo. Many embryonic abnormalities related to shortness in the neural tube such as spina bifida, encephalocele, and anencephaly has been proven in the past years $[2,4]$. The growth of the central nervous system has a direct correlation with the growth and survival of an individual. A shortage in the evolution of neural cells leads to deficiencies in the natural performance of these cells from the viewpoint of discharge of cerebrospinal fluid and other materials necessary for the evolution of the central nervous system $[5,6]$. The role of endocrine glands, including the thyroid gland, in the body's metabolic activities is an undeniable reality. The effects of hyperthyroidism or hypothyroidism and related hormonal fluctuations can have an in- 
tensive impact on the mechanism of the body's chemical interactions [7]. A delay in the growth of the brain and the cerebellum can happen because of hyperthyroidism or hypothyroidism $[8,9]$. Any factor which causes a delay in the natural evolution of nervous cells will cause a disruption in the natural performance of nervous cells, and this deficiency can create many abnormalities in the nervous system, including microcephaly $[10,11]$. The trend of brain evolution in rodents is a very good model for studying the action of thyroid hormones in the central nervous system $[11,12]$. The best animal model of brain growth dependent on thyroid hormones is found in the infants of field rats [13]. Hyperthyroidism induction in animals is usually performed with the two methods of genetic induction and using medicine which can be planned based on the type and target of the study. An embryo's brain growth in the beginning of pregnancy depends on the mother's thyroid hormone. Non-treatment of hyperthyroidism in pregnant women causes a high risk for pregnancy poisoning, embryonic heart failure, lack of sufficient embryonic growth, preterm delivery, and embryonic death inside the womb. The greatest state of hyperthyroidism is in the form of Graves' disease. Untreatedhyperthyroidism in pregnant mothers is associated with hyperthyroidism in embryos $[14,15]$.

\section{Objectives}

As thyroid hormones play key roles in the evolution of different systems, hyperthyroidism will create undeniable evolutionary deficiencies in the nervous system [16]. To date, the effects of hyperthyroidism on the evolution of the parietal lobe have not been studied from a histology point of view; the present research investigated this subject.

\section{Methods}

Approval for this experimental study was obtained from the Institutional Review Board, and all experiments were carried out in accordance with the guidelines of animal care and use of the ethics committee of Baqiyatallah University of Medical Sciences. Thirty mature female Sprague-Dawley field rats with a weight limit of $200 \pm 10$ grams were obtained from the Pasteur institute of Iran. The rats were placed in a controlled environment with a temperature of $23 \pm 2^{\circ} \mathrm{C}$, humidity of $50 \pm 5 \%$, and 12 hours of light and 12 hours of dark per day. To allow them to acclimate to the environment, all male and female rats were kept for two weeks separately in the animal house of Baqiyatallah University of Medical Sciences and were fed with standard food. The rats were randomly divided into three groups. The control group which received no materials remained intact. The sham group received a distilled water solution with common salt and polysorbate, and the experimental group received intraperitoneal injections of 0.5 $\mathrm{mg} / \mathrm{kg}$ levothyroxine during a 10-day period. All rats were weighed and blood was taken was done through the sinuous inside of the eye orbit. Two weeks after the beginning of injections, blood was again taken from all groups. To determine the rates of T3, T4, and TSH hormones, the samples were sent to the endocrinology laboratory at Taleghani hospital. A gamma counter and R.I.A. kits were used to measure T4 and T3, and an IRMA kit was used to measure TSH.

After definite detection of hyperthyroidism in the rats of the experimental group, the rats in all groups were mated. Day zero of pregnancy was determined in the pregnant rats of the experimental group, and throughout the pregnancy, IP injections of T4 were made daily; the rats of the sham group received daily injections of distilled water solution containing polysorbate and Nacl.

At the end of day 20 of pregnancy, the pregnantrats were killed by inhalation of chloroform in a high dose. Initially, blood samples were taken from each mother rat and sent to the lab. Then the embryos were separated from inside the uterine horns, and the weight of the embryos and the crown-rump length (CRL) of the embryos were measured and registered. Finally, the embryos were placed inside a container containing $10 \%$ formalin for fixation. Five embryos out of the embryos of each pregnant mouse were randomly selected. After texture processing, sections measuring 5 microns thick were prepared, stained with the haematoxylin-Eosin ( $\mathrm{H}$ and $\mathrm{E}$ ) method, and evaluated by optical microscope and Motic software. The data was reviewed using the ANOVA method and Tukey test in SPSS 22 software. The information was presented in the form of mean $\pm S E M$, and a difference in means was considered significant at the level of $\mathrm{P}<0.05$.

\section{Results}

During the tests, it became apparent that the embryos in both the control and sham groups were fully healthy and lacked any kind of morphological abnormality. The rate of T4 serum in the hyperthyroid mothers was about $265.8 \pm 3.2$ millimolar per liter, and in the control mothers it was $62.7 \pm 2.3$ millimolar per liter. The variables under examination in the mothers and embryos of the control and sham groups showed no significant difference in any case.

The results showed that the mean CRL length in the experimental group was significantly reduced compared with the control and sham groups (Figure 1). 
Figure 1. Comparison of Mean CRLs of Embryos in the Control, Sham, and Experimental Groups

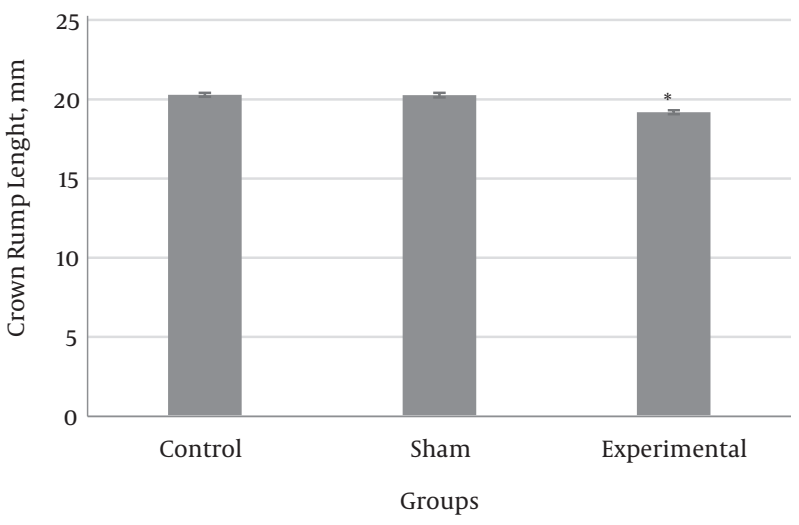

The sign * shows the presence of a significant reduction between the experimental and the control and sham groups $(\mathrm{P}<0.05)$.

The mean weight of embryos in the experimental group showed no significant difference compared with the control and sham groups $(\mathrm{P}<0.05)$ (Figure 2$)$.

Figure 2. The Mean Embryo Weights in the Control,Sham, and Experimental Groups

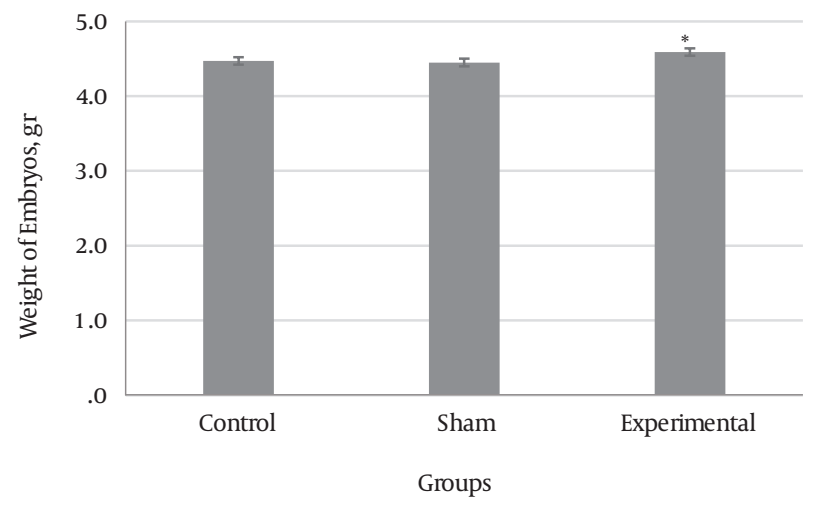

The sign * shows the presence of anincrease between he experimental group and the control and sham groups $(\mathrm{P}<0.05)$.

The results showed that the total thickness of the parietal cortex and the thickness of the cortical, intermediate, and ventricular layers in the experimental group as compared with the control and sham groups had a reduction; however, this reduction was insignificant only in the cortical layer (Figure 3).

According to Figure 4, the number of cells in all three layers of the texture of the brain parietal cortex showed no significant difference between the control and sham groups. In all three layers, a difference is seen between the experimental group and the control and sham groups which indicates a reduction in the number of cells. However, only in the ventricular layer is there a significant difference between the experimental group and the control and sham groups $(\mathrm{P}<0.05)$.

\section{Discussion}

Research has shown that, in addition to embryonic thyroid hormones, the maternal thyroid hormones are also necessary for the natural growth of the central nervous system before birth. A shortage of thyroid hormones during the pre-birth period will lead to irreversible damage to the brain and mental retardation [14]. Madeira et al. (1991) reviewed the impacts of hyperthyroidism on the granular layer of the dentate gyrus of the hippocampus in mature male and female rats. They concluded that a shortage of thyroid hormones causes a reduction of neuronal multiplication and increase of cell death [17]. Maternal hyperthyroidism can be a factor in increasedembryonicabortion and absorption, reduction of infant brain size and weight, and increasedembryonic mortality.

The rate of $\mathrm{T} 4$ serum in hyperthyroid mothers (experimental $265.8 \pm 3.2)$ and in the control mothers $(62.7 \pm 2.3$ $\mathrm{nmol} / \mathrm{L}$ ) had a significant difference. The research of Azizi et al. showed that the T4 and T3 serum levels were significantly higher in the group of hyperthyroid patients than in the control group [18]. The findings of the present study indicated a significant increase in the serum levels of T4 thyroid hormones, in agreement with Azizi et al. During pregnancy in humans and rats, the maternal thyroid hormones, in particular the Tiroxin hormone (T4), are transferred to the embryo through the placenta. The T4 hormone is changed into its active form, i.e. T3, after its transfer into the embryonic brain or the removal of iodine from it. If the rate of these hormones is at a natural level, the embryo develops naturally; a decrease or increase in the rate of thyroid hormones of the mother can be a factor of disruption of growth in embryos $[19,20]$. The fluctuations related to each hormone can intensively influence the mechanism of body chemical interactions [21]. The mother's thyroid hormones, particularly in the first half of pregnancy, are significantly important for the evolution of the embryo [22].

In the present research, the weight of embryos in the experimental group compared with those in the control and sham groups had a significant increase. These results are in agreement with the findings of Freidoun Azizi et al. in 2003 who showed that maternal hyperthyroidism causes the acceleration of osseous cells and increases the activity of osteoblast cells in embryos. It seems that the increase of embryonic weight in the experimental group of this study was due to the increased rate of osteoblast. The 


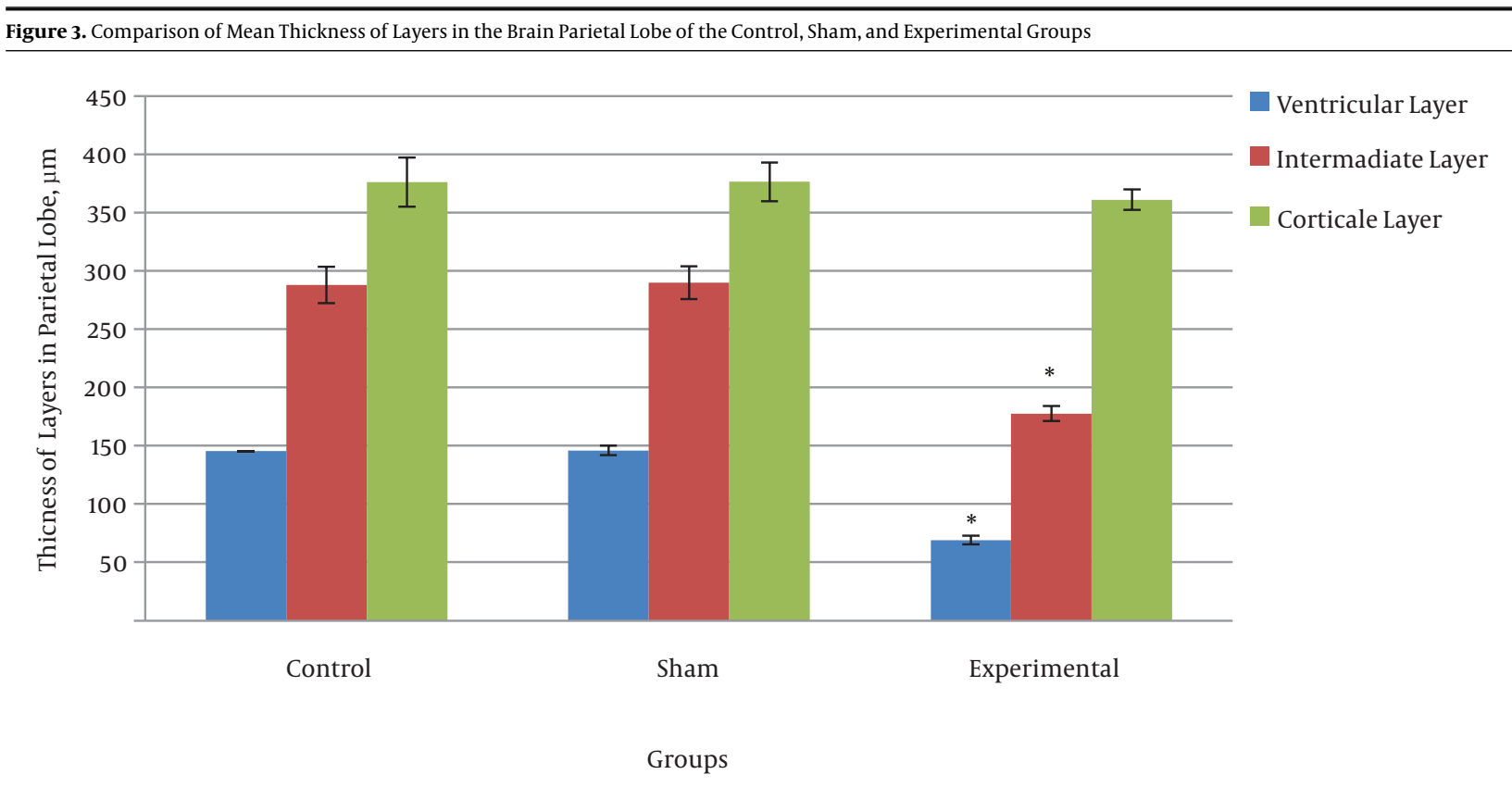

The sign * shows a significant reduction toward the control and sham groups $(\mathrm{P}<0.05)$.

length of long bones and their mineralized part, in particular in the bones of upper organs in the embryos of the experimental group, showed that maternal hyperthyroidismand the passage of thyroid hormones through the placenta caused the acceleration of osseous growth and increase dosteoblast cells in embryos [18].

The results of the present research showed that maternal hyperthyroidism causes a significant reduction in CRL of the embryos in the experimental group compared with the control group. In astudy by Freidoun Azizi et al., the size of CRL between embryos of the experimental and control groupswas not significantly different, and these results are in agreement with the findings of the current study [18]. In the present research, the mother's hyperthyroidismhad no impact on the weight and size of the placenta in the control and experimental groups. In the study of Freidoun Azizi et al., the weight and length of the placenta in embryos of the experimental and control groups did not show a significant difference, and these results are in agreement with the current findings [18]. It seems that the placenta in rats acts as an obstacle restricting the transfer of T3 [13].

The results obtained from the concerned parameters in this research indicated a kind of significant reduction in the thickness of the forming layers of the brain parietal cortex and a reduction in the number of their cells. In fact, it can be said that the impact of maternal hyperthyroidism induced with the maximum dose of levothyroxine led to a reduction in growth. The experimental groups on average showed a reduction in the thickness of layers and a reduction in the number of cells compared with the control group. The results of histomorphometry and cell counting in a study showed that the mother's affliction with hyperthyroid disease during pregnancy controlled cell multiplication in the external granular layer of the cerebellum and diminished the neural migration in the inner layer towards the side of the cortical area [23, 24]. It seems that maternal hyperthyroidism also causes a delay to occur in embryonic brain growth, because the shortage of thyroid hormone in the embryotic period causes unnatural growth and a lack of distinction in cerebellum cells. This issue was also observed in rodents, i.e. the impact of the thyroid hormone on the trend of evolution of the cerebellum by two weeks after birth [20]. If the thyroid hormones do not discharge sufficiently in the embryonic period, the natural evolution of the cerebellum will face difficulty [25, 26].These results are in agreement with the findings of the current study.

\subsection{Conclusion}

The present study showed that maternal hyperthyroidism during pregnancy can have a negative impact on the evolution of the central nervous system and cause a reduction in growth of the CNS in the embryos of these mothers. Maternal hyperthyroidism leads to the emergence of many embryonic abnormalities and deficiencies. 
Figure 4. Cell Counting in Cortical, Intermediate, and Ventricular Layers of Parietal lobe in Control, Sham, and Experimental Groups (Resolution X400, H and E Staining)
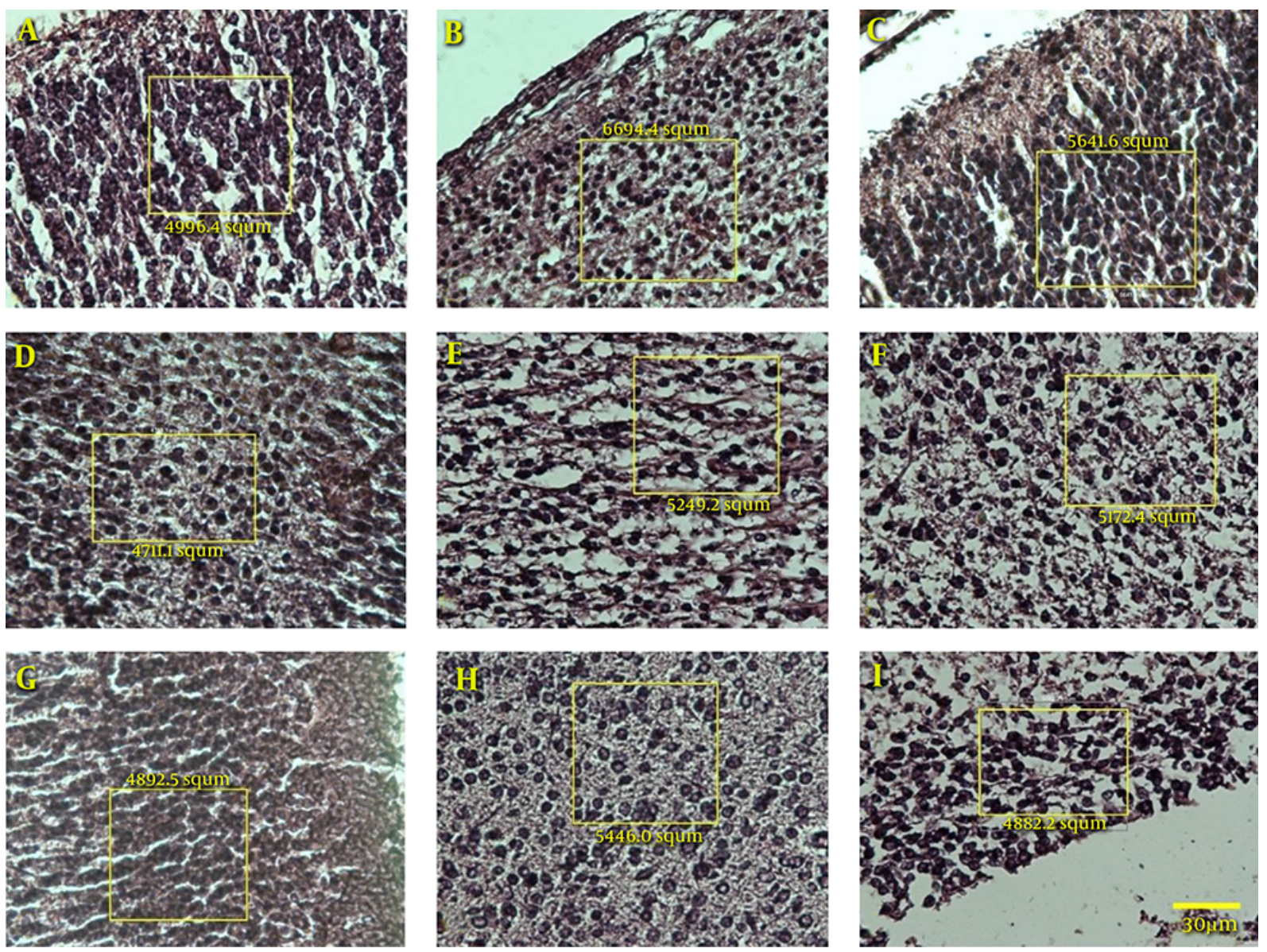

A - C, cortical layer; D - F, intermediate layer; G - I, ventricular layer. A, D, G, control group; B, E, H, sham group; C, F, I, experimental group.

This study confirms well the impact of maternal hyperthyroidism on organogenesis, in particular in embryonic growth and evolution of the central nervous system.

\section{Acknowledgments}

This article is the result of research in the neurosciences research center at Baqiyatallah University of Medical Sciences. The authors hereby express their sincere appreciation to this center.

\section{Footnotes}

Authors' Contribution: Fatemeh Mirsafi, animal care, surgery of the animals, and study design; Mahnaz Azarnia, histopathologic study; Gholamreza Kaka, data analysis and writing.
Conflict of Interests: The authors have no conflicts of interest.

Funding/Support: None declared.

\section{References}

1. Sadler TW. Langman's medical embryology. Lippincott Williams \& Wilkins; 2011.

2. Boyles AL, Billups AV, Deak KL, Siegel DG, Mehltretter L, Slifer SH, et al. Neural tube defects and folate pathway genes: family-based association tests of gene-gene and gene-environment interactions. Environ Health Perspect. 2006;114(10):1547-52. doi: 10.1289/ehp.9166. [PubMed: 17035141].

3. Korevaar TI, Muetzel R, Medici M, Chaker L, Jaddoe VW, de Rijke $\mathrm{YB}$, et al. Association of maternal thyroid function during early pregnancy with offspring IQ and brain morphology in childhood: a population-based prospective cohort study. Lancet Diabetes Endocrinol. 2016;4(1):35-43. doi: 10.1016/S2213-8587(15)00327-7. [PubMed: 26497402].

4. Arum J, Azarnia M, Kaka G, Sadraei SH, Kamalinejad M. Study of elaeagnus angustifolia fruit aqueous extract on the histomorphometrical 
changes of retina in mouse embryo. Zahedan J Res Med Sci. 2016;18(10) doi:10.17795/zjrms-3865.

5. Moreno-Manzano V, Rodriguez-Jimenez FJ, Garcia-Rosello M, Lainez S, Erceg S, Calvo MT, et al. Activated spinal cord ependymal stem cells rescue neurological function. Stem Cells. 2009;27(3):733-43. doi: 10.1002/stem.24. [PubMed: 19259940].

6. Neman J, Chen TC. The Choroid Plexus and Cerebrospinal Fluid. 59. Neuroscience; 2007. pp. 745-57.The Choroid Plexus.

7. Nandi-Munshi D, Taplin CE. Thyroid-related neurological disorders and complications in children. Pediatr Neurol. 2015;52(4):373-82. doi: 10.1016/j.pediatrneurol.2014.12.005. [PubMed: 25661286].

8. Ahmed OM, El-Gareib AW, El-Bakry AM, Abd El-Tawab SM, Ahmed RG. Thyroid hormones states and brain development interactions. Int $J$ Dev Neurosci. 2008;26(2):147-209. doi: 10.1016/j.ijdevneu.2007.09.011. [PubMed: 18031969].

9. El-Bakry AM, El-Gareib AW, Ahmed RG. Comparative study of the effects of experimentally induced hypothyroidism and hyperthyroidism in some brain regions in albino rats. Int $J$ Dev Neurosci. 2010;28(5):371-89. doi: 10.1016/j.ijdevneu.2010.04.003. [PubMed: 20412848].

10. Ulrich CM, Potter JD. Folate supplementation: too much of a good thing?. Cancer Epidemiol Biomarkers Prev. 2006;15(2):189-93. doi: 10.1158/1055-9965.EPI-152CO. [PubMed: 16492904].

11. Andersen SL, Laurberg P, Wu CS, Olsen J. Maternal thyroid dysfunction and risk of seizure in the child: a Danish nationwide cohort study. J Pregnancy. 2013;2013:636705. doi: 10.1155/2013/636705. [PubMed: 23984072].

12. Ahmed OM, Ahmed RG, El-Gareib AW, El-Bakry AM, Abd El-Tawab SM. Effects of experimentally induced maternal hypothyroidism and hyperthyroidism on the development of rat offspring: II-the developmental pattern of neurons in relation to oxidative stress and antioxidant defense system. Int J Dev Neurosci. 2012;30(6):517-37. doi: 10.1016/j.ijdevneu.2012.04.005. [PubMed: 22664656].

13. Desouza LA, Sathanoori M, Kapoor R, Rajadhyaksha N, Gonzalez LE, Kottmann AH, et al. Thyroid hormone regulates the expression of the sonic hedgehog signaling pathway in the embryonic and adult Mammalian brain. Endocrinology. 2011;152(5):1989-2000. doi: 10.1210/en.2010-1396. [PubMed: 21363934].

14. Van Herck SL, Geysens S, Bald E, Chwatko G, Delezie E, Dianati E, et al. Maternal transfer of methimazole and effects on thyroid hormone availability in embryonic tissues. J Endocrinol. 2013;218(1):105-15. doi: 10.1530/JOE-13-0089. [PubMed: 23608220].

15. Irles C, Nava-Kopp AT, Moran J, Zhang L. Neonatal maternal separation up-regulates protein signalling for cell survival in rat hypothalamus. Stress. 2014;17(3):275-84. doi: 10.3109/10253890.2014.913017. [PubMed: 24730533].

16. Amerion M, Houshmand Z, Tahajjodi SS, Mahdavi Shahri N, Jalali
M, Nikravesh MR. The immunohistochemical effect of maternal hypothyroidism and hyperthyroidism during pregnancy and lactation on skin development in wistar rat newborns [In Persian].J Cell Tissue. 2012;3(1):1-8.

17. Madeira MD, Cadete-Leite A, Andrade JP, Paula-Barbosa MM. Effects of hypothyroidism upon the granular layer of the dentate gyrus in male and female adult rats: a morphometric study. J Comp Neurol. 1991;314(1):171-86. doi: 10.1002/cne.903140116. [PubMed: 1797872].

18. Azizi F, Raiszadeh F, Solati M, Etemadi A, Arabi M, Rahmani M. Serum lipids, lipoproteins, apolipoproteins and paraoxanase enzyme activity in patients with thyroid dysfunction [In Persian]. Iran J Endocrinol Metab. 2001;3(1):11-22.

19. Lisboa PC, Oliveira E, Fagundes AT, Santos-Silva AP, Conceicao EP, Passos MC, et al. Postnatal low protein diet programs leptin signaling in the hypothalamic-pituitary-thyroid axis and pituitary TSH response to leptin in adult male rats. Horm Metab Res. 2012;44(2):114-22. doi: 10.1055/s-0031-1299747. [PubMed: 22314332].

20. Ahmed OM,Abd El-Tawab SM, Ahmed RG. Effects of experimentally in duced maternal hypothyroidism and hyperthyroidism on the development of rat offspring: I. The development of the thyroid hormonesneurotransmitters and adenosinergic system interactions. Int J Dev Neurosci. 2010;28(6):437-54. doi: 10.1016/j.ijdevneu.2010.06.007. [PubMed: 20599606].

21. Kar A, Panda S, Bharti S. Relative efficacy of three medicinal plant extracts in the alteration of thyroid hormone concentrations in male mice. J Ethnopharmacol. 2002;81(2):281-5. doi: 10.1016/S03788741(02)00048-X. [PubMed: 12065164].

22. Zhang L, Medina MP, Hernandez VS, Estrada FS, Vega-Gonzalez A. Vasopressinergic network abnormalities potentiate conditioned anxious state of rats subjected to maternal hyperthyroidism. Neuroscience. 2010;168(2):416-28. doi:10.1016/j.neuroscience.2010.03.059. [PubMed: 20371268].

23. Sampson D, Pickard M, Evans I, Leonard A, Sinha A, Ekins R. Thyroid hormone regulates the expression of alpha-internexin in neurons in culture. Neuroreport. 2002;13(3):273-6. doi: 10.1097/00001756200203040-00005. [PubMed: 11930121].

24. Sampson D, Pickard MR, Sinha AK, Evans IM, Leonard AJ, Ekins RP. Maternal thyroid status regulates the expression of neuronal and astrocytic cytoskeletal proteins in the fetal brain. $J$ Endocrinol. 2000;167(3):439-45. doi:10.1677/joe.0.1670439. [PubMed: 11115770].

25. Bernal J. Thyroid hormone receptors in brain development and function. Nat Clin Pract Endocrinol Metab. 2007;3(3):249-59. doi: 10.1038/ncpendmet0424. [PubMed: 17315033].

26. Zoeller RT, Rovet J. Timing of thyroid hormone action in the developing brain: clinical observations and experimental findings. J Neuroendocrinol. 2004;16(10):809-18. doi:10.1111/j.1365-2826.2004.01243.x. [PubMed: 15500540]. 